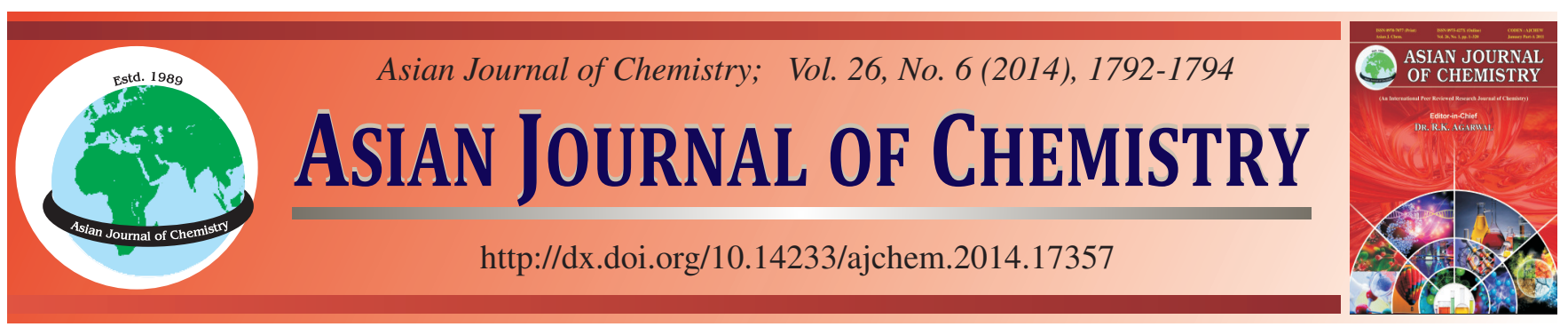

\title{
Electrophoretic Deposition Fabrication and Magnetic Manipulation of Magnetic Semiconductor Films $\dagger$
}

\author{
Liting Zhang ${ }^{1}$, Jianjun Li ${ }^{1,2, *}$, Jinbo Zhu ${ }^{1}$, Yin LiU ${ }^{1}$, Fanfei Min ${ }^{1}$ and Weichang HaO ${ }^{2}$
}

${ }^{1}$ Department of Materials Science and Engineering, Anhui University of Science and Technology, Huainan 232001, P.R. China

${ }^{2}$ Center of Materials Physics and Chemistry, Beihang University, Beijing 100083, P.R. China

*Corresponding author: E-mail: lijj3@aust.edu.cn

Published online: 10 March 2014;

AJC-14898

\begin{abstract}
ZnO:Co semiconductor films were synthesized by a two-step electrophoretic deposition method. Various morphologies, including solid, crystal-grain or nanorod-array film, could be obtained by tuning the deposition conditions. Careful characterizations indicate the morphologies and structures have great effect on the magnetic and optical properties of the films. Significant difference of magnetism was investigated in the films prepared under different deposition potentials. The origination of the magnetism difference and the mechanism of the electrophoretic deposition were discussed.
\end{abstract}

Keywords: Magnetic semiconductor films, Electrophoretic deposition, Nanorod arrays, Magnetism difference.

\section{INTRODUCTION}

Ferromagnetic semiconductors (FMSs) have attracted great interests for their potential applications in semiconductor spintronics devices ${ }^{1}$. Cobalt doped $\mathrm{ZnO}$ has been predicted to be one of the most promising candidate ferromagnetic semiconductors for achieving room-temperature Curie temperature and thus, it has been receiving great attentions in recent years ${ }^{2}$. $\mathrm{Zn}_{1-\mathrm{x}} \mathrm{Co}_{\mathrm{x}} \mathrm{O}$ thin films have been fabricated via various methods, including pulsed laser deposition, magnetron co-sputtering, molecular-beam epitaxy, chemical vapor deposition, ultrasonicassisted solution, ion-beam sputtering and sol-gel method. Both intrinsic and extrinsic ferromagnetism have been experimentally observed ${ }^{3,4}$. There is no conclusion on which deposition method is best for achieving ferromagnetic ordering films. The magnetic behaviours of the films are found sensitive to the deposition conditions ${ }^{5-9}$. Therefore, controllable tuning of the morphologies and structures of the films is of great importance.

Electrophoretic deposition method is a two-step process for film fabrication and nano-structure synthesis ${ }^{10-13}$. In a traditional electrophoretic deposition, precursor colloidal is carefully prepared, which acts as the working fluid. Under the action of electric field, electrically charged colloidal particles in the colloidal moved to certain electrode and deposition on the substrate or in the template holes. After subsequent treatments, thin film or micro-structural materials are obtained. In this work, $\mathrm{Zn}_{0.95} \mathrm{Co}_{0.05} \mathrm{O}$ thin films were fabricated via $\mathrm{DC}$ electrophoretic deposition method under different deposition potential and time. Carefully characterizations indicate the obtained films have different morphology and structure. Magnetic investigation shows that they are of room-temperature ferromagnetic. The mechanism of electrophoretic deposition is discussed.

\section{EXPERIMENTAL}

All the reagents used in this experiment are of analytical grade, purchased from Shanghai Sinopharm Chemical Reagent Co. Ltd. The Si(111) substrate used in this work is $99.999 \%$ in purity. The substrates were first soaked in boiling sulfuric acid $(98 \%)$ for $1 \mathrm{~h}$, then rinsed ultrasonically in deionized water and finally dried in a vacuum oven. The water used in all process is tri-distilled water.

Experiments and characterizations: The $\mathrm{Zn}_{0.95} \mathrm{Co}_{0.05} \mathrm{O}$ films were grown on $\mathrm{Si}$ (111) substrates by an electrophoretic deposition method. Electrophoretic deposition was performed in a tri-electrodes system, using $\mathrm{Zn}(\mathrm{OAc})_{2}: \mathrm{Co}(\mathrm{OAc})_{2}$ composite colloid as electrophoretic solution. An Si(111) slice $(2 \mathrm{~cm} \times 4$ $\mathrm{cm})$, acted as working electrode (negative electrode) and a reeled Pt thread acted counter electrode. The electrophoretic 
deposition is a two-step process. A direct potential of $4.5 \mathrm{~V}$ was applied on the electrodes and sustained $90 \mathrm{~s}$ for the first step deposition. After 100 s quick annealing, a $\mathrm{ZnO}$ seeds film was obtained. For the second deposition, a direct potential of 5.0, 6.5 and $8.0 \mathrm{~V}$ was applied on the electrodes and sustained for $45 \mathrm{~min}$, the corresponding films were named as F50, F65 and F80, respectively. Then the prepared samples were annealed at $500{ }^{\circ} \mathrm{C}$ for $6 \mathrm{~h}$ in air atmosphere.

The scanning electron microscopy (SEM) images were obtained by a JSM-5600LV SEM equipped with energy dispersive spectrometer (EDS). The structure variations were characterized by using Rigaku D/Max-2200 XRD with $\mathrm{CuK}_{\alpha}(1.54 \AA$ ) line. The magnetic properties were characterized by VSM (LakerShore 7410) at $300 \mathrm{~K}$.

\section{RESULTS AND DISCUSSION}

SEM images (Fig. 1a-c) show the surface and the crosssection (the inset patterns) morphologies of the $\mathrm{Zn}_{0.95} \mathrm{Co}_{0.05} \mathrm{O}$ films. It is seen that the thicknesses of F50, F65 and F80 are $2.63,6.39$ and $7.24 \mu \mathrm{m}$, respectively. The thickness is increasing with the electrophoretic potential, which leads to the deference of the deposition rate. The surface morphologies of the films are obviously different. The dense degree of F65 film is obviously lower than that of the F80, but higher than that of F50. In fact, from the cross-section patterns, it could be found that the F50 film is composed of isolated nanorods. It has also been proved by the investigation of the transmission electronic microscopy (not shown). The EDS spectrum (Fig. 1d) suggests that the films are only composed of $\mathrm{Zn}$, Co and $\mathrm{O}$ elements. The atomic ratio of $\mathrm{Zn}$ to Co is measured as 81:4, very close to the nominal Co doping ratio.
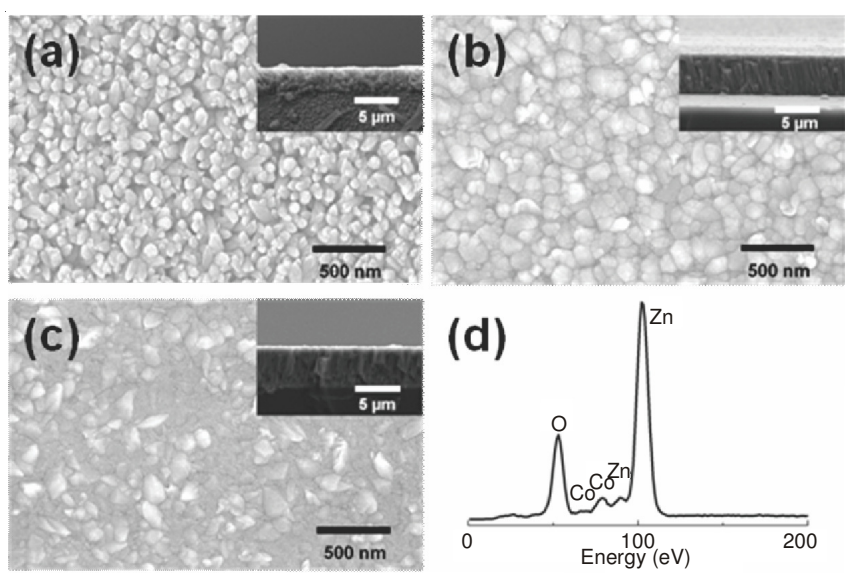

Fig. 1. (a)-(c) the surface SEM images of F50, F65 and F80 films, respectively. The insert shows their corresponding cross-section images. (d) The EDS spectra of the F50 film

Fig. 2a shows the XRD spectra of the $\mathrm{Zn}_{0.95} \mathrm{Co}_{0.05} \mathrm{O}$ films, the spectrum of a $\mathrm{Si}(111)$ substrate is also present for comparison. All the $\mathrm{Zn}_{0.95} \mathrm{Co}_{0.05} \mathrm{O}$ films are found of hexagonal structure similar to that of Wurtzite ZnO (JCPDS number: \#65-3411). There are only one peak at $34.26^{\circ}$ in all the XRD patterns, which indicates the deposited films have complete (002) preferred growth. The FWHM (full width at half maximum peak) of the F50 (002) peak is much higher than that of the other two for the formation of the isolated nanorods. According

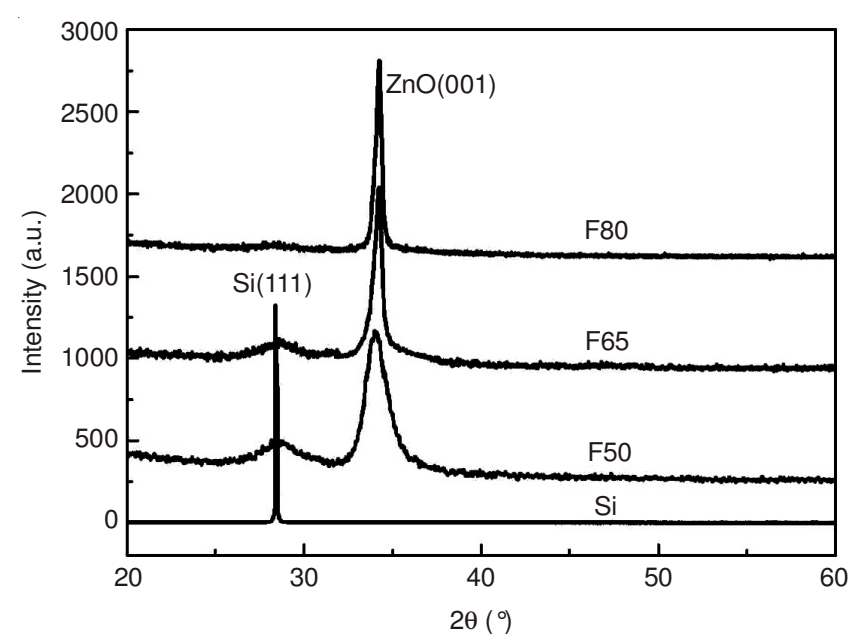

Fig. 2. XRD spectra of F50, F65, F80 films and Si(111) substrate

to the Sherrer formula, the diameter of the nanorods is estimated to be $35-40 \mathrm{~nm}$, close to the SEM and TEM results. The diffraction peak at $28.4^{\circ}$ are corresponding to the $\mathrm{Si}(111)$ crystal plane. Its intensity decreases with the increase of the electrophoretic potential for the decreasing of the thickness and dense degree.

Fig. 3 shows the magnetization of the $\mathrm{Zn}_{0.95} \mathrm{Co}_{0.05} \mathrm{O}$ films measured by VSM at room temperature. All the samples show room-temperature ferromagnetism. The magnetisms of F80 and F65 are $2.593 \times 10^{-3}$ and $6.702 \times 10^{-3} \mathrm{emu} / \mathrm{cm}^{3}$, respectively. While the magnetism of F50 is much higher, to reach $15.961 \times 10^{-3} \mathrm{emu} / \mathrm{cm}^{3}$. It is believed that in $\mathrm{ZnO}$ : Co system, bound magnetic polarons (BMPs) model plays an important role in mediating the ferromagnetic interaction ${ }^{14,15}$. Therefore, in the same $\mathrm{Zn}_{0.95} \mathrm{Co}_{0.05} \mathrm{O}$ system, the magnetism should be in a similar scale. The significant difference of magnetism, especially for the sharp magnetism increasing of F50, should be originated from the structural difference of the films. The perceptible coercivity of F50 is smaller than that of F65 and F80, as shown in the inset of Fig. 4.

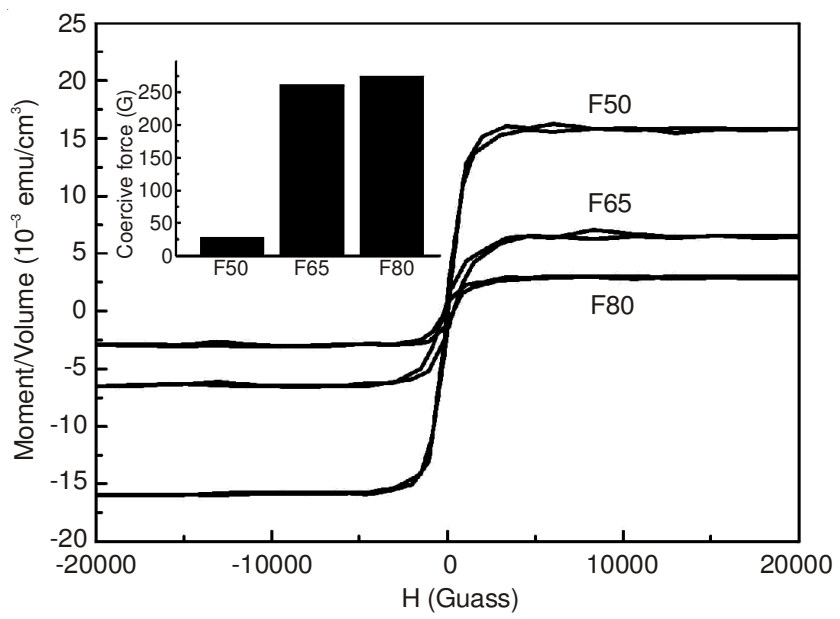

Fig. 3. M-H curves and the coercivety (the inset pattern) of F50, F65, F80 films detected by VSM at $300 \mathrm{~K}$

The microstructure of the $\mathrm{Zn}_{0.95} \mathrm{Co}_{0.05} \mathrm{O}$ films is closely related to the electrophoretic parameters. At the beginning of the electrophoretic deposition, the $\mathrm{ZnO}$ : Co colloidal particles 
with a little adsorbed liquid moved to cathode (Si substrate with crystal seeds) when the external electric field was applied to the electrodes. Then, there present a high $\mathrm{ZnO}$ : Co concentration area near the Si substrate surface. Under the induction of the crystal seeds, the colloidal particles deposited and finally formed the film. The $\mathrm{ZnO}$ : Co concentration near the substrate is decided by the electrophoretic potential, if other parameters keep unchanged. If the precursor concentration is too high, the deposition rate is out of control of the induction of the crystal seeds. As a result, solid films formed, just as the F80 film. Furthermore, in such a rapid growth, some of the Co ions will not properly doped in $\mathrm{ZnO}$ crystal seeds second-phase $\mathrm{Co}_{3} \mathrm{O}_{4}$ present. Only in a merely supersaturation condition the induction of the crystal seeds is dominant, then nanorods arrays formed, just as the F50 film.

\section{Conclusion}

$\mathrm{ZnO}$ :Co semiconductor films with various structures, including solid film, crystal-grain film and nanorod-array films, were controllably synthesized by an electrophoretic deposition method. Careful investigations indicated the morphologies, structures and magnetisms of the films were closely related to the electrophoretic deposition potential. The mechanism of electrophoretic deposition was also discussed.

\section{ACKNOWLEDGEMENTS}

This work is supported by the National Natural Science Foundation of China (Grant No. 51208003) and China Postdoctoral Science Foundation (Grant No. 1208085ME84).

\section{REFERENCES}

1. S. Wolf, D. Awschalom, R. Buhrman, J. Daughton, S. Von Molnar, M. Roukes, A. Chtchelkanova and D. Treger, Science, 294, 1488 (2001).

2. T. Dietl, H. Ohno, F. Matsukura, J. Cibert and D. Ferrand, Science, 287, 1019 (2000).

3. H. Gu, Y. Jiang, Y. Xu and M. Yan, Appl. Phys. Lett., 98, 012502 (2011).

4. B.B. Straumal, A.A. Mazilkin, S.G. Protasova, A.A. Myatiev, P.B. Straumal, E. Goering and B. Baretzky, Thin Solid Films, 520, 1192 (2011).

5. K. Samanta, P. Bhattacharya, R. Katiyar, W. Iwamoto, P. Pagliuso and C. Rettori, Phys. Rev. B, 73, 245213 (2006).

6. L.J. Zhang, J.Q. Wang, J. Li, J. Zhou, W.P. Cai, J. Cheng, W. Xu, G. Yin, X. Wu, Z. Jiang, S. Zhang and Z.-Y. Wu, Chem. Commun., 48, 91 (2011).

7. R. Ranjan, H.S. Nabi and R. Pentcheva, J. Appl. Phys., 105, 053905 (2009).

8. Y. Peng, D. Huo, H. He, Y. Li, L. Li, H. Wang and Z. Qian, J. Magn. Magn. Mater., 324, 690 (2012).

9. D. Seghier and H. Gislason, J. Mater. Sci. Mater. Electron., 22, 1400 (2011).

10. I. Corni, M.P. Ryan and A.R. Boccaccini, J. Eur. Ceram. Soc., 28, 1353 (2008).

11. Y. Wang and I. Zhitomirsky, Langmuir, 25, 9684 (2009).

12. J. Li, L. Zhang, J. Zhu, Y. Liu, W. Hao and B. Li, Mater. Lett., 87, 101 (2012).

13. P. Koidl, Phys. Rev. B, 15, 2493 (1977).

14. J.M.D. Coey, A. Douvalis, C.B. Fitzgerald and M. Venkatesan, Appl. Phys. Lett., 84, 1332 (2004).

15. G. Ice, Nat. Mater., 4, 17 (2005). 